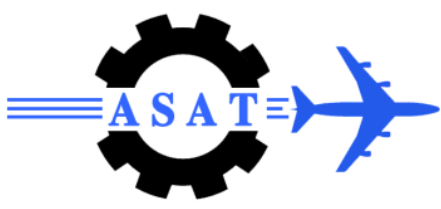

\title{
Numerical Solution of Inviscid Transonic Flow Using Hybrid Finite Volume-Finite Difference Solution Technique on Unstructured Grid
}

\author{
M. B. Azab* , M. I. Mustafa ${ }^{\dagger}$
}

\begin{abstract}
An explicit cell centered finite volume CFD solver called Pyramid2D has been developed for solving Euler's equations in two dimensions. We modify the flow equations at the boundary faces, according to the physical boundary conditions, and solve them in finite difference discrete form suitable for unstructured grids. The flow properties at the cell faces are constructed using new reconstruction method which allows small oscillations at the shock wave region and in the same time contains enough diffusion, so it doesn't require the use of limiters for transonic flows. The developed solver is to be used as an external aerodynamic solver; all tests showed that the proposed scheme is robust and its numerical results compares well with the published data.
\end{abstract}

\section{Introduction}

The improvement of computer's computational power and the development of state of the art numerical simulation techniques encourage aerospace engineers to use computational fluid dynamics in aerodynamic design and analysis. Numerical solution of fluid flow using finite volume method has been well established during the last three decades [1]. Finite volume method is very popular solution technique for aerodynamic simulation problems using unstructured grids because it takes the advantage of unstructured grid ability to present complex geometry efficiently. Boundary conditions are applied by controlling the boundary face flux that crosses it according to the physical boundary conditions at that boundary face; however, flow property values at the boundaries are computed using the extrapolation of flow property values of the internal flow points. This leads to an incomplete satisfaction of the boundary conditions especially at solid surface boundaries; therefore an algebraic correction is applied to the extrapolated flow properties to satisfy the physical boundary conditions. This extrapolation usually tends to increase the required number of iterations to reach a steady state solution. In the other hand, finite difference aerodynamic simulations applies the boundary conditions to the discrete form of the governing equations, this leads to more accurate satisfaction of the boundary conditions and needs no algebraic correction[2]. The standard finite difference discretization needs structured grids to be implemented, which in turn needs multi-blocking gridding strategy in order to present complex geometries and requires huge programming effort. In this paper we present a new CFD strategy that takes the advantage of finite volume as a discretization method for the internal flow field control volumes, and the

\footnotetext{
Advanced Numerical Simulation Laboratory, the University Of British Columbia, Vancouver, Canada.

${ }^{\dagger}$ Egyptian Armed Forces, Egypt
} 
accurate satisfaction of the boundary conditions obtained by using finite difference at the boundary faces mid points. We implement $2^{\text {nd }}$ order finite volume discretization of Euler's $2 \mathrm{D}$ flow model for the internal flow points, and we use a new treatment of the boundary faces using finite differences on 2D unstructured triangular grid. Results show that the proposed scheme contains sufficient diffusion and hence there is no need for using limiters in transonic flow simulations. In section two, we present the basics of the cell centered finite volume scheme on unstructured triangular mesh. The face property reconstruction needed for Roe's approximate Riemann solver to evaluate the flux at cell faces is presented in section three. The new developed finite difference discretization on unstructured grid is applied at the boundary faces as shown in section four. In section five we present three test cases to test the developed solver, transonic flow around NACA 0012 where the shock wave exist on upper and lower surfaces of the airfoil, a transonic flow around NACA 0012 where the shock stands at the airfoil trailing edge, and supersonic flow around NACA 0012 with detached shock wave in front of the airfoil leading edge. All test cases are done without the use of limiter, results shows good agreement with published numerical results.

\section{Explicit Finite Volume Solver}

The integral form of the two dimensional Euler's equations can be written as,

$$
\frac{\partial}{\partial t} \iint Q \cdot d V+\oint_{d \Omega} \vec{F} \cdot \hat{n} \cdot d l=0
$$

where $\hat{n}=n_{x} i+n_{y} j$ is the outward pointing normal to the control volume faces, and

$$
\begin{aligned}
& Q=\left[\begin{array}{c}
\rho \\
\rho \cdot u \\
\rho \cdot v \\
E_{t}
\end{array}\right], \vec{F} \cdot \hat{n}=\left[\begin{array}{c}
\rho \cdot U \\
\rho \cdot U \cdot u+n_{x} \cdot p \\
\rho \cdot U \cdot v+n_{y} \cdot p \\
\left(E_{t}+p\right) \cdot U
\end{array}\right], \\
& p=(\gamma-1) \cdot\left[E_{t}-\rho \cdot \frac{\left(u^{2}+v^{2}\right)}{2}\right], \\
& U=n_{x} u+n_{y} v
\end{aligned}
$$

Figure (1) shows a schematic drawing of a triangular element and its immediate neighbours. Equation (1) can be rewritten as,

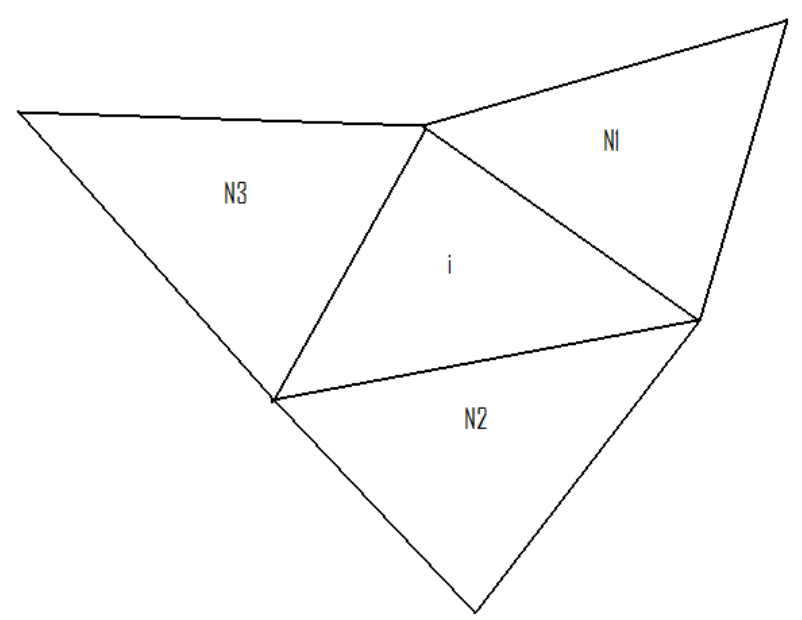

Figure (1): schematic drawing of an unstructured triangular element with its neighbors 
$\frac{A_{i}}{\Delta t} \cdot \Delta Q=R$

where

$R=-\sum_{N i} F_{N i} \cdot l_{N i}=-F_{N 1} \cdot l_{N 1}-F_{N 2} \cdot l_{N 2}-F_{N 3} \cdot l_{N 3}$

$F_{N i}$ is the flux that goes across the face between element $\mathrm{i}$ and its neighbour $\mathrm{Ni} ; l_{N i}$ is the face length of the face between the element and its neighbour.

The face flux is computed using Roe flux differencing scheme [3].

The flow properties at control volume center point is updated as follows,

$Q^{n+1}=Q^{n}+\Delta Q$

\section{Face Flow Property Reconstruction}

In order to evaluate the face fluxes, primitive flow properties $q=\left\{\begin{array}{llll}\rho & u & v & p\end{array}\right\}^{T}$ must be constructed at the mid points of the element faces. The flow properties at the faces are calculated using the following $2^{\text {nd }}$ order accurate formula,

$q_{f}=q_{i}+\frac{\partial q}{\partial x} \cdot \Delta x_{i f}+\frac{\partial q}{\partial y} \cdot \Delta y_{i f}+O(\Delta)^{2}$

where $\Delta x_{i f}, \Delta y_{i f}$ are the $\mathrm{x}$ and $\mathrm{y}$ distances between the center of element $\mathrm{i}$, and the face midpoint. The gradient components $\frac{\partial q}{\partial x} \cdot \frac{\partial q}{\partial y}$ are computed as follows,

Let $q=a_{0}+a_{1} x+a_{2} y$

$\therefore \frac{\partial q}{\partial x}=a_{1}$

$\ldots \frac{\partial q}{\partial y}=a_{2}$

Writing equation (5) at the three vertices of the element (i), shown in Figure (2), gives the following linear system,

$$
\left[\begin{array}{ccc}
1 & x_{a} & y_{a} \\
1 & x_{b} & y_{b} \\
1 & x_{c} & y_{c}
\end{array}\right] \cdot\left\{\begin{array}{l}
a_{0} \\
a_{1} \\
a_{2}
\end{array}\right\}=\left\{\begin{array}{l}
q_{a} \\
q_{b} \\
q_{c}
\end{array}\right\}
$$

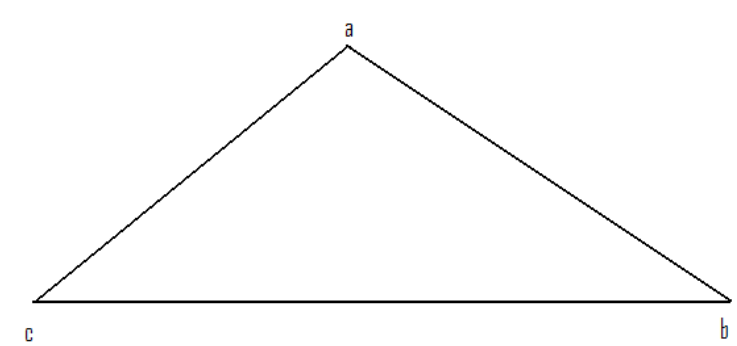

Figure (2): Vertices names in an element

The coefficients $a_{0}, a_{1}$ and $a_{2}$ are found by solving the system of linear equations (7), and hence, find $\frac{\partial q}{\partial x}$ and $\frac{\partial q}{\partial y}$ needed for face property reconstruction as of equation (4). 
The face reconstructed flow properties from both sides are used to compute the face flux according to Roe flux difference formula $[3,4]$ as follows,

$$
\begin{aligned}
& F_{f}=\frac{1}{2}\left[F\left(Q_{R}\right)+F\left(Q_{L}\right)-|\tilde{A}| \cdot\left(Q_{R}-Q_{L}\right)\right] \\
& F_{f}=\frac{1}{2}\left[F\left(Q_{R}\right)+F\left(Q_{L}\right)-\left|\Delta F_{1}\right|-\left|\Delta F_{4}\right|-\left|\Delta F_{5}\right|\right]
\end{aligned}
$$

where all the terms of equation (9) are expressed as of Neal Frink et al. [4].

The nodal properties are found using inverse distance interpolation technique [4]. The following inverse distance formula is used,

$$
q_{n}=\frac{\sum_{1}^{m} \frac{1}{r_{c i}} q_{c i}}{\sum_{1}^{m} \frac{1}{r_{c i}}}
$$

Figure (3) shows a general node in triangular mesh grid, in this figure $m=6$ "the number of elements including a node".

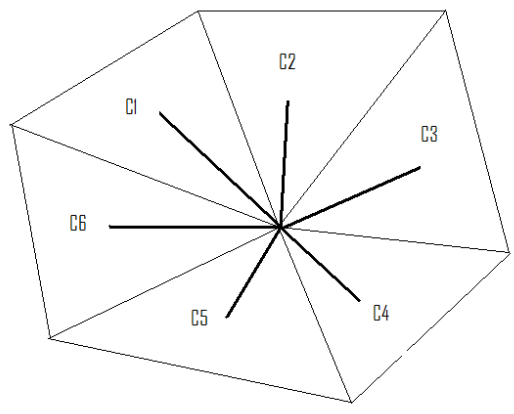

Figure (3): Schematic drawing of a node included in 6 elements

If the node is a boundary node, the inverse distance formula, equation (10) is applied to the boundary faces that share this boundary node and other elements that share this node are neglected when using the inverse distance interpolation rule.

The above scheme for nodal property reconstruction provides some diffusion in the face reconstruction scheme, and hence, a stable solution can be obtained in transonic flows without the use of limiters.

\section{Finite Difference on Unstructured Grids}

Euler's equations for two dimensional compressible flows can be written as,

$\frac{\partial Q}{\partial t}+\frac{\partial E}{\partial x}+\frac{\partial G}{\partial y}=0$

where,

$$
\begin{aligned}
& Q=\left[\begin{array}{c}
\rho \\
\rho . u \\
\rho . v \\
E
\end{array}\right], \quad E=\left[\begin{array}{c}
\rho . u \\
\rho . u^{2}+p \\
\rho . u \cdot v \\
\left(E_{t}+p\right) \cdot u
\end{array}\right]=\left[\begin{array}{c}
E_{1} \\
E_{2} \\
E_{3} \\
E_{4}
\end{array}\right], \quad G=\left[\begin{array}{c}
\rho \cdot v \\
\rho \cdot u \cdot v \\
\rho . u .^{2}+p \\
\left(E_{t}+p\right) \cdot v
\end{array}\right]=\left[\begin{array}{c}
G_{1} \\
G_{2} \\
G_{3} \\
G_{4}
\end{array}\right] \\
& p=(\gamma-1) \cdot\left[E_{t}-\rho \cdot \frac{\left(u^{2}+v^{2}\right)}{2}\right]
\end{aligned}
$$


Traditional finite difference forms are expressed on structured grids where the neighbours are known by the structured grid indexing stencil. The spatial derivatives $\frac{\partial}{\partial x}, \frac{\partial}{\partial y}$ can be expressed easily in structured grids. The challenge in using finite difference on unstructured grids is how to find a suitable expression of the spatial derivatives $\frac{\partial}{\partial x}, \frac{\partial}{\partial y}$. To do so, differentiation chain rule is used as follows,

$$
\begin{aligned}
& \frac{\partial E}{\partial x}= \frac{\partial E}{\partial q} \cdot \frac{\partial q}{\partial x} \\
& \frac{\partial E}{\partial x}=\left[\begin{array}{llll}
\frac{\partial E_{1}}{\partial q_{1}} & \frac{\partial E_{1}}{\partial q_{2}} & \frac{\partial E_{1}}{\partial q_{3}} & \frac{\partial E_{1}}{\partial q_{4}} \\
\frac{\partial E_{2}}{\partial q_{1}} & \frac{\partial E_{2}}{\partial q_{2}} & \frac{\partial E_{2}}{\partial q_{3}} & \frac{\partial E_{2}}{\partial q_{4}} \\
\frac{\partial E_{3}}{\partial q_{1}} & \frac{\partial E_{3}}{\partial q_{2}} & \frac{\partial E_{3}}{\partial q_{3}} & \frac{\partial E_{3}}{\partial q_{4}} \\
\frac{\partial E_{4}}{\partial q_{1}} & \frac{\partial E_{4}}{\partial q_{2}} & \frac{\partial E_{4}}{\partial q_{3}} & \frac{\partial E_{4}}{\partial q_{4}}
\end{array}\right] \cdot\left[\begin{array}{l}
\frac{\partial q_{1}}{\partial x} \\
\frac{\partial q_{2}}{\partial x} \\
\frac{\partial q_{3}}{\partial x} \\
\frac{\partial q_{4}}{\partial x}
\end{array}\right],
\end{aligned}
$$

where $\frac{\partial E}{\partial q}$ depends only on flow properties and known as "primitive variables flux jacobian", and $\frac{\partial q}{\partial x}$ is computed using the procedure shown in section (3). The same strategy can be applied to compute $\frac{\partial G}{\partial y}$.

\section{Boundary Conditions}

Boundary conditions treatments play an important role in obtaining a converged solution. In this paper the presented boundary condition treatments are based on the work of M. B. Azab et al., [2] in which the boundary conditions are used instead of some flow equations at the boundary points, the rest of the flow equations in addition to the boundary conditions equations are solved in a coupled manner in order to obtain the flow properties at the boundary points which lie on the boundary surfaces. Both equations are solved in a finite difference discrete form, while the rest flow field solution is obtained using finite volume scheme. In external aerodynamics, the two important boundary conditions are the far field inflow/outflow and the wall boundary conditions. Numerical treatments of the boundary conditions are shown in the next subsections.

\subsection{Far Field Inflow}

All flow properties are specified at the far field inflow boundary, flow properties are equal to free stream values; therefore the finite difference form of Euler's equation can be written as, $\frac{\Delta Q}{\Delta t}=\left[\begin{array}{l}0 \\ 0 \\ 0 \\ 0\end{array}\right]$

The far field inflow is the far field surface in which,

$$
U_{n}=n_{x} \cdot u_{\infty}+n_{x} \cdot u_{\infty}<0
$$




\subsection{Far Field Outflow}

Only the back pressure is specified at the boundary face to be equal to the free stream static pressure $P=P_{\infty}$, the following manipulation is used in order to derive the boundary condition numerical treatment in a FD form,

$$
\begin{aligned}
& P=P_{\infty} \\
& \frac{\partial P}{\partial t}=\frac{\partial P}{\partial Q_{1}} \cdot \frac{\partial Q_{1}}{\partial t}+\frac{\partial P}{\partial Q_{2}} \cdot \frac{\partial Q_{2}}{\partial t}+\frac{\partial P}{\partial Q_{3}} \cdot \frac{\partial Q_{3}}{\partial t}+\frac{\partial P}{\partial Q_{4}} \cdot \frac{\partial Q_{4}}{\partial t}=0 \\
& \frac{\Delta P}{\Delta t}=\frac{\partial P}{\partial Q_{1}} \cdot \frac{\Delta Q_{1}}{\Delta t}+\frac{\partial P}{\partial Q_{2}} \cdot \frac{\Delta Q_{2}}{\Delta t}+\frac{\partial P}{\partial Q_{3}} \cdot \frac{\Delta Q_{3}}{\Delta t}+\frac{\partial P}{\partial Q_{4}} \cdot \frac{\Delta Q_{4}}{\Delta t}=0 \\
& \therefore \Delta Q_{4}=\frac{1}{\left(\frac{\partial P}{\partial Q_{4}}\right)} \cdot\left\{\frac{\partial P}{\partial Q_{1}} \cdot \Delta Q_{1}+\frac{\partial P}{\partial Q_{2}} \cdot \Delta Q_{2}+\frac{\partial P}{\partial Q_{3}} \cdot \Delta Q_{3}\right\}, \\
& \therefore \Delta Q_{4}=\lambda_{14} \cdot \Delta Q_{1}+\lambda_{24} \cdot \Delta Q_{2}+\lambda_{34} \cdot \Delta Q_{3}, \\
& \text { where, } \lambda_{i 4}=\frac{\partial P}{\partial Q_{i}} / \frac{\partial P}{\partial Q_{4}}, \\
& P=(\gamma-1) \cdot\left(Q_{4}-\frac{1}{2} \cdot\left(\frac{Q_{2}^{2}+Q_{3}^{2}}{Q_{1}}\right)\right) .
\end{aligned}
$$

Using the above equations, the FD equations at the boundary face will be,

$$
\frac{1}{\Delta t} \cdot\left[\begin{array}{l}
\Delta Q_{1} \\
\Delta Q_{2} \\
\Delta Q_{3} \\
\Delta Q_{4}
\end{array}\right]=-\left[\begin{array}{c}
\frac{\partial E_{1}}{\partial x} \\
\frac{\partial E_{2}}{\partial x} \\
\frac{\partial E_{3}}{\partial x} \\
\lambda_{14} \frac{\partial E_{1}}{\partial x}+\lambda_{24} \frac{\partial E_{2}}{\partial x}+\lambda_{34} \frac{\partial E_{3}}{\partial x}
\end{array}\right]^{n}-\left[\begin{array}{c}
\frac{\partial G_{1}}{\partial y} \\
\frac{\partial G_{2}}{\partial y} \\
\frac{\partial G_{3}}{\partial y} \\
\lambda_{14} \frac{\partial G_{1}}{\partial y}+\lambda_{24} \frac{\partial G_{2}}{\partial y}+\lambda_{34} \frac{\partial G_{3}}{\partial y}
\end{array}\right]^{n},
$$

The far field outflow is the far field surface in which,

$U_{n}=n_{x} \cdot u_{\infty}+n_{x} \cdot u_{\infty} \geq 0$

Using equation (17), the solution update is computed and the flow solution at the boundary face is marched temporally as,

$Q^{n+1}=Q^{n}+\Delta Q$

\subsection{Inviscid Wall}

The physical boundary condition for inviscid wall is that the normal velocity to the wall is zero, which can be written as,

$$
\begin{aligned}
& U_{n}=\vec{n} \cdot \vec{U} \\
& U_{n}=n_{x} \cdot u+n_{y} \cdot v=0,
\end{aligned}
$$


$\therefore n_{x} \cdot \rho u+n_{y} \cdot \rho v=0$

$n_{x} \cdot \Delta Q_{2}+n_{y} \cdot \Delta Q_{3}=0$

The boundary condition equation (21) will replace one of the momentum equations. The implemented strategy is to drop the momentum equation ( $\mathrm{x}$ or y momentum) which is closer to normal direction; in other words, if $n_{y}>n_{x}$ the y-momentum equation will be dropped and vice versa.

In case of $n_{y}>n_{x}$, the FD equations at the wall face will be,

$$
\frac{1}{\Delta t} \cdot\left[\begin{array}{c}
\Delta Q_{1} \\
\Delta Q_{2} \\
\Delta Q_{3} \\
\Delta Q_{4}
\end{array}\right]=-\left[\begin{array}{c}
\frac{\partial E_{1}}{\partial x} \\
\frac{\partial E_{2}}{\partial x} \\
\frac{-n_{x}}{n_{y}} \cdot \frac{\partial E_{2}}{\partial x} \\
\frac{\partial E_{4}}{\partial x}
\end{array}\right]^{n}-\left[\begin{array}{c}
\frac{\partial G_{1}}{\partial y} \\
\frac{\partial G_{2}}{\partial y} \\
\frac{-n_{x}}{n_{y}} \cdot \frac{\partial G_{2}}{\partial y} \\
\frac{\partial G_{4}}{\partial y}
\end{array}\right]^{n},
$$

And in case of $\mathrm{n}_{\mathrm{x}}>\mathrm{n}_{\mathrm{y}}$ the FD equations will be,

$$
\frac{1}{\Delta t} \cdot\left[\begin{array}{c}
\Delta Q_{1} \\
\Delta Q_{2} \\
\Delta Q_{3} \\
\Delta Q_{4}
\end{array}\right]=-\left[\begin{array}{c}
\frac{\partial E_{1}}{\partial x} \\
\frac{-n_{y}}{n_{x}} \cdot \frac{\partial E_{3}}{\partial x} \\
\frac{\partial E_{3}}{\partial x} \\
\frac{\partial E_{4}}{\partial x}
\end{array}\right]^{n}-\left[\begin{array}{c}
\frac{\partial G_{1}}{\partial y} \\
\frac{-n_{y}}{n_{x}} \cdot \frac{\partial G_{3}}{\partial y} \\
\frac{\partial G_{3}}{\partial y} \\
\frac{\partial G_{4}}{\partial y}
\end{array}\right]^{n},
$$

The computed solution update resulting from equation (22) or (23) is used to update the conservative flow properties at the boundary face according to equation (19).

\section{Test Cases}

In this section, three test cases are presented, flow around NACA0012 at Mach 0.8 and angle of attack $1.25^{\circ}$, a highly transonic flow around NACA 0012 at Mach 0.95 and angle of attack $0^{\circ}$, and supersonic flow around NACA 0012 at $\mathrm{Mach}=1.5$ and zero angle of attack. Comparison with published AGARD [5] data shows good agreement.

\subsection{Transonic NACA 0012 Flow $\left(\mathrm{M}=0.8\right.$, Alfa $\left.=1.25^{\circ}\right)$}

In this test case, transonic flow solution of NACA 0012 at Mach 0.8 and $1.25^{\circ}$ angle of attack is obtained using the developed solver with the new boundary condition treatments. Figure (5) shows a comparison between Pyramid2D numerically obtained $\mathrm{Cp}$ and the published AGARD Cp distribution; a slight deviation is observed at the leading edge region and is attributed to the difference of the leading edge grid resolution between the structured grid used in AGARD published data and the unstructured grid used in Pyramid2D computations. Figure (5) shows also an accurate prediction of the shock waves on the upper and lower airfoil 
surfaces. Figure (5) shows also that there is a slight undershooting in the obtained pressure distribution at the shock wave position but this oscillation is not observed elsewhere. As these results were obtained without the use of a limiter, it proves that the proposed face property scheme together with the node property inverse distance interpolation produce sufficient diffusion at the shock wave region to damp oscillation propagation to other regions.

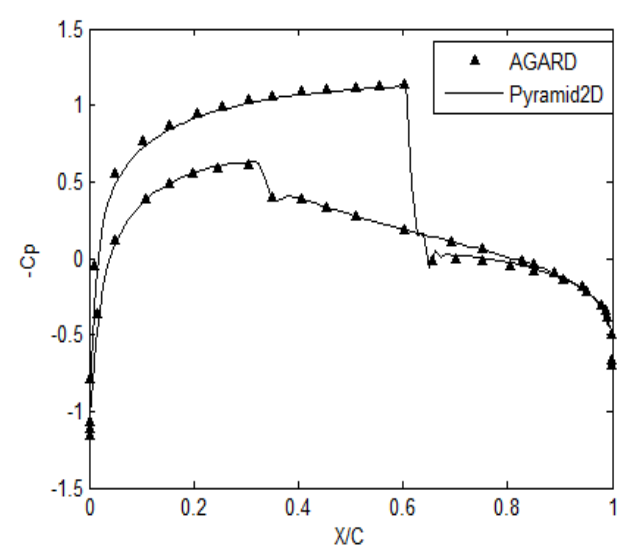

Figure (5): NACA0012 Cp distribution comparison with AGARD published data at $\mathrm{Mach}=0.8$, Alfa=1.25

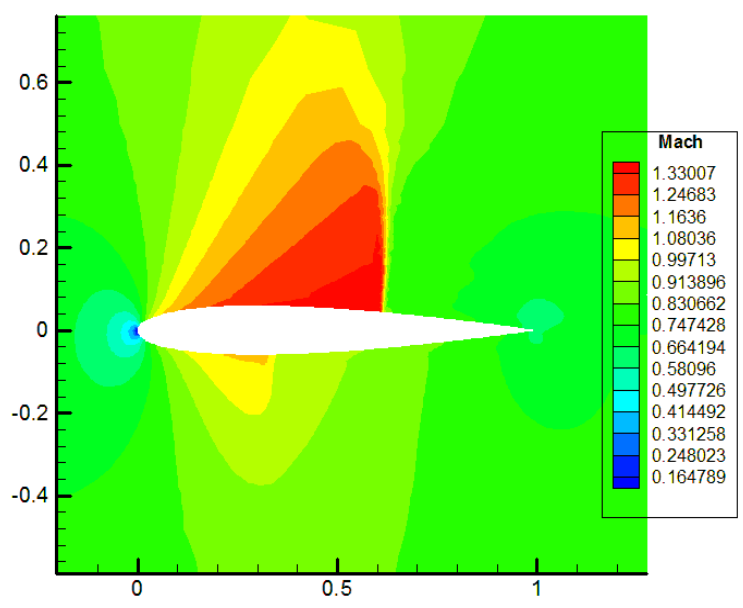

Figure (6): NACA0012 Mach distribution at free stream Mach=0.8, Alfa=1.25

\subsection{Transonic NACA 0012 Flow $\left(\mathrm{M}=0.95\right.$, Alfa $\left.=0^{\circ}\right)$}

In this test case, a continuous expansion all over the airfoil surface after the leading edge stagnation point followed by a strong oblique shock wave standing at the airfoil trailing edge is observed as shown in figure (8). Figure (7) shows a comparison of the obtained Cp distribution and the $\mathrm{Cp}$ distribution published in AGARD; it shows a very good agreement between both $\mathrm{Cp}$ distributions.

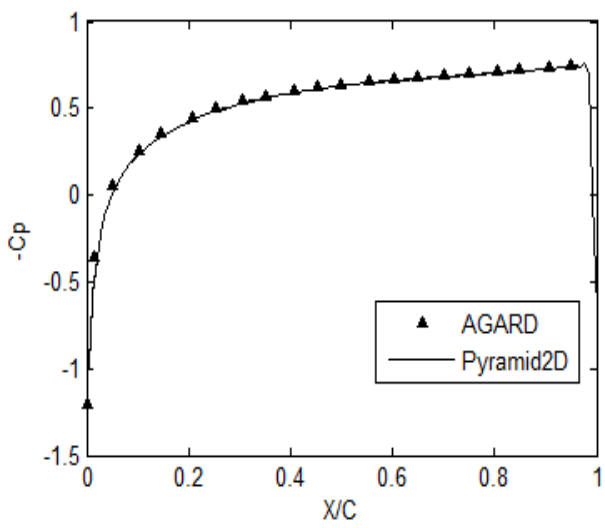

Figure (7): NACA0012 Cp distribution comparison with AGARD published data at Mach $=0.95$, Alfa $=0$

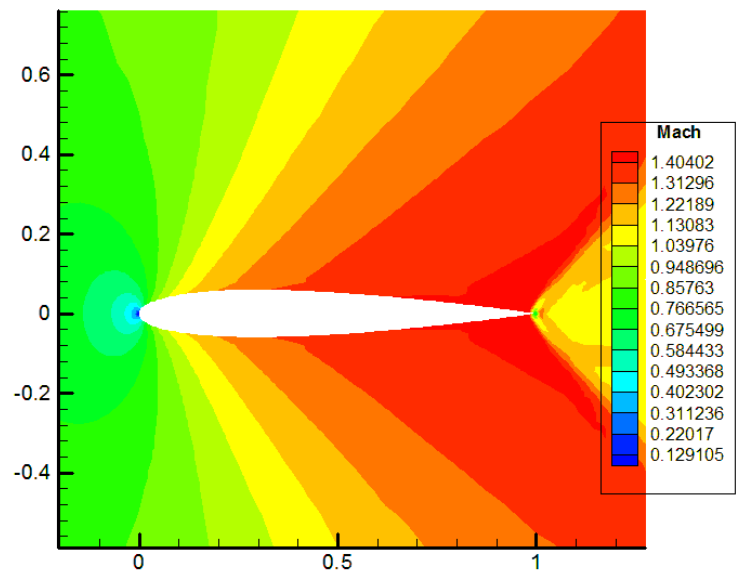

Figure (8): NACA0012 Mach distribution at free stream Mach $=0.95$, Alfa $=0$

\subsection{Supersonic NACA 0012 Flow $\left(\mathrm{M}=1.5\right.$, Alfa $\left.=0^{\circ}\right)$}

In this test case, a strong detached shock wave is formed ahead of the airfoil leading edge decreasing the flow speed to subsonic regime ; a rapid expansion along the airfoil upper and lower surfaces accelerate the flow to supersonic conditions flowed by an oblique shock wave 
at the trailing edge as shown in figure (9). No published data for this test case is available for the authors, therefore no comparison is presented.

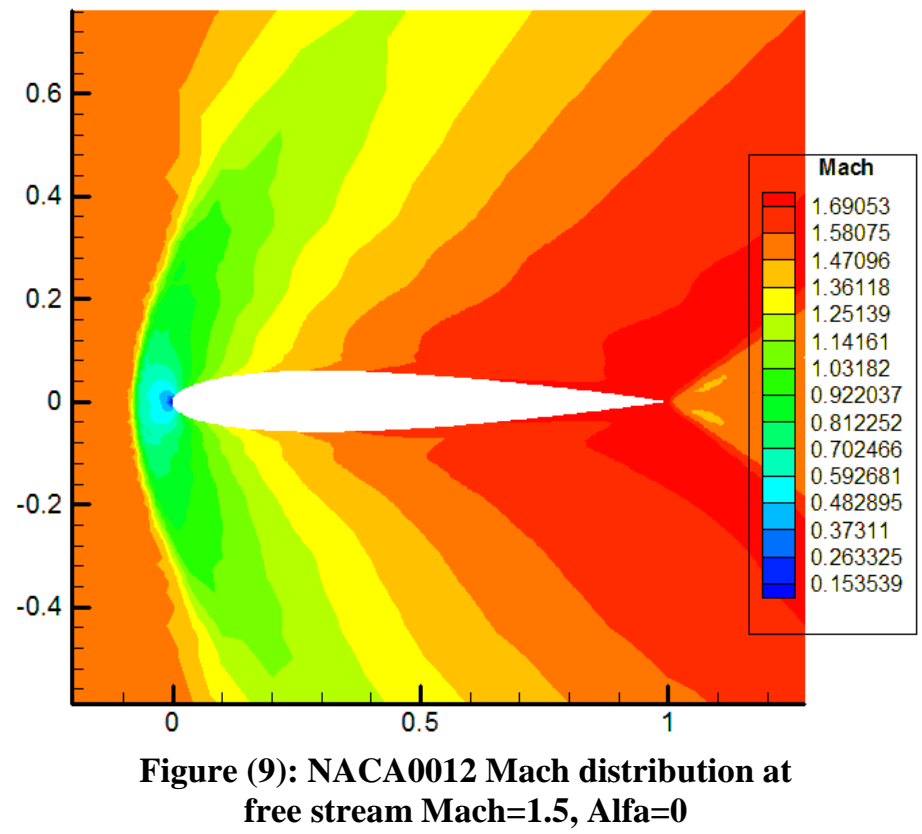

\section{Conclusions}

New successful property face reconstruction scheme has been presented. The presented method together with inverse distance based interpolation for nodal property reconstruction produces sufficient diffusion in the shock wave region such that there is no need for limiter usage. The proposed boundary conditions treatment was successful and presented accurate results matching AGARD published data. The new hybrid finite volume/finite difference solver successfully converged to machine zero in all the test cases; extension to three dimensions is straight forward.

\section{Acknowledgement}

Authors want to acknowledge Prof. Dr. Carl Ollivier-Gooch, the University of British Columbia, Vancouver Canada, for his valuable remarks and discussions.

\section{References}

1 Jameson, A. A Perspective on Computational Algorithms for Aerodynamic Analysis and Design; Progress in Aerospace Sciences, 2001, 37(2), 197-243.

2 Azab, M.B., Abdelrahman, M. M., Mostafa, M. I., On the Numerical Solution of the Inviscid Two Dimensional Internal Flows with Non-Orthogonal Grids, $12^{\text {th }}$ International Conference on Aerospace Sciences \& Aviation Technology, Military Technical College, Cairo, Egypt, 2007, CFD-03.

3 Roe, P.L. Approximate Riemann Solvers, Parameter Vectors, and Difference Schemes, Journal of Computational Physics, 1981, 43(2), 357-372.

4 Frink N. T., P.P., Pirzadeh S., A Fast Upwind Solver for the Euler equations on ThreeDimensional Unstructured Meshes, AIAA paper 91-0102, 1991.

5 NATO, Test Cases for Inviscid Flow Field Methods, AGARD Advisory Report No 211. 